\title{
Genome assembly of Fusarium oxysporum f. sp. lini using a combination of Oxford Nanopore and Illumina reads
}

\author{
Dvorianinova E.M. ${ }^{1,2 *}$, Novakovskiy R.O. ${ }^{1}$, Pushkova E.N. ${ }^{1}$, Kudryavtseva L.P. ${ }^{3}$, Rozhmina T.A. ${ }^{1,3}$, \\ Krasnov G.S. ${ }^{1}$, Melnikova N.V. ${ }^{1}$, Dmitriev A.A. ${ }^{1}$ \\ ${ }^{1}$ Engelhardt Institute of Molecular Biology, Russian Academy of Sciences, Moscow, Russia \\ ${ }^{2}$ Moscow Institute of Physics and Technology, Dolgoprudny, Russia \\ ${ }^{3}$ Federal Research Center for Bast Fiber Crops, Torzhok, Russia \\ *e-mail: dvorianinova.em@phystech.edu
}

Key words: flax pathogens, $F$. oxysporum f. sp. lini, genome assembly, Nanopore, Illumina

Motivation and Aim: Fusarium oxysporum f. sp. lini is regarded as one of the most harmful flax pathogens, causing its wilt. Since it is of high significance to study this species at molecular and genetic levels, its genome sequence will be of great help in such a field of investigations. Thus, our aim was to achieve a high-quality genome assembly of $F$. oxysporum $\mathrm{f}$. sp. lini pathogenic isolate \#39. We used a combination of Nanopore and Illumina sequencing platforms that allow one to obtain long and high accuracy reads respectively.

Methods and Algorithms: F. oxysporum pathogenic isolate \#39 was provided by the Institute for Flax (Torzhok, Russia) and grown on potato dextrose agar for 3 weeks. Pure high-molecular DNA was extracted from fungal mycelium according to our developed protocol. The assessment of quality and quantity of the extracted nucleic acid was performed by the methods of fluorometry (Qubit 2.0), spectrophotometry (Nanodrop), and electrophoresis in $0.8 \%$ agarose gel. DNA libraries were prepared and sequenced on Illumina (HiSeq 2500 instrument, HiSeq Rapid SBS Kit V2, 2×250 bp) and Nanopore (MinION instrument, FLO-MIN-106 R9.4 flow-cell) platforms according to the manufacturers' protocols. Nanopore reads were bacecalled using guppy 3.2.2, and low-quality ones were filtered out using trimmomatic 0.38 . The initial assembly was performed using Canu 1.8 and polished twice. Firstly, we used Racon 1.4.3 and Medaka 0.12.1 for polishing with Nanopore reads and then POLCA for assembly correction with filtered and trimmed Illumina reads.

Results: After sequencing, we managed to achieve 110x coverage in total - 80x for Nanopore reads and $30 \mathrm{x}$ for Illumina data. According to BUSCO, the completeness of the final assembly appeared to be $99.5 \%$, while QUAST statistics were the following: total length of $59 \mathrm{MB}, \mathrm{N} 50$ of $3.3 \mathrm{MB}, 35$ contigs.

Conclusion: The combination of data received from the two sequencing platforms allowed us to obtain a high-quality assembly of $F$. oxysporum $\mathrm{f}$. sp. lini genome, the knowledge of which will support further investigations of $F$. oxysporum and flax-pathogen interaction. The assembled genome can be accessed at DDBJ/ENA/GenBank (accession - WHMS00000000, BioProject accession - PRJNA578147).

Acknowledgements: Supported by RSF (16-16-00114, genome sequencing and assembly) and RFBR (1934-90055, the development of a protocol for DNA extraction). 